\title{
EDITORIAL
}

\section{Helping the homogeneous}

\author{
H.E. Fessler and D. Feller-Kopman
}

$\mathbf{T}$ he National Emphysema Treatment Trial (NETT) [1] helped clarify the patient selection criteria and expected risks and benefits of lung volume reduction surgery (LVRS) for patients with advanced emphysema. The NETT built on numerous earlier case series which showed that the greatest symptomatic and functional benefit accrued to patients with a predominantly upper lobe distribution of emphysema. In such patients who also had very low exercise tolerance, the surgery lowered their mortality risk over the next 5 yrs by about half. Importantly for patients in the USA, findings from the study were used to establish the criteria for Medicare coverage of the procedure. This opened the door for thousands of qualifying patients to undergo this life-enhancing and life-extending procedure.

However, in the 6 yrs since, barely a trickle of emphysema patients has crawled through that door. In 2007 and 2008, only about a dozen lung volume reduction procedures per month were billed through Medicare (personal communication, J. Baldwin, Centers for Medicare and Medicaid Services). The reasons for this discrepancy are speculative, but could include concerns about the small but non-trivial operative mortality, the substantial morbidity of major surgery, the impermanence of improvement, and the difficulty predicting benefit for an individual despite propitious characteristics. Patients and their physicians have been making the intensely personal decision that the benefit of this surgery is not worth the risk. For patients whose emphysema is not in the upper lobe predominant distribution (about half of the patients enrolled in the NETT), the calculus is even less favourable.

To redress this risk-benefit balance, a profusion of creative bronchoscopic approaches to the mechanical derangements of emphysema are under investigation. One method, primarily being studied for patients with diffuse, or "homogeneous" disease, creates holes, supported by drug-eluting stents, directly from segmental bronchi into the surrounding parenchyma. This "airway bypass" allows decompression of underventilated space-occupying cysts and bullae. Gas trapped at a distance from the fenestrations can still find egress via the low resistance collateral channels present in emphysema. This method has shown modest benefits in pulmonary function, but has been handicapped by closure of the holes. The optimal size, number and location of the holes, as well as the safety of the procedure, remain to be determined [2].

Pulmonary and Critical Care Medicine, Johns Hopkins School of Medicine, Baltimore, MD.

CORRESPONDENCE: H.E. Fessler, Division of Pulmonary and Critical Care, 1830 Monument St 5th Floor, Baltimore, MD 21287, USA. E-mail: hfessler@jhmi.edu
Other methods use bronchoscopically placed one-way (expiratory) valves to promote atelectasis of distal lung regions. These studies have also shown some modest benefits in lung function or symptoms, along with some complications such as pneumothorax and prolonged air leak [3, 4]. To generalise regarding these valve systems, the risks appear to be much lower than surgical lung volume reduction, but the gains in lung function are also smaller. Furthermore, it remains challenging to predict the benefit for any individual patient. The limiting factor in this method may be the same low collateral resistance that forms the basis for the airway bypass technique. With the valves, collateral ventilation prevents absorptive atelectasis and thereby limits the improvement in lung mechanics. Methods for measuring collateral resistance to optimise patient selection or valve placement are also being developed and commercialised [5]. Thus far, however, for many ingenious bronchoscopic variations of LVRS, attractively low risk has come with disappointingly low benefit.

Other investigational bronchoscopic approaches to lung volume reduction may overcome the impediment of collateral ventilation. One uses steam to create a controlled burn and scar in the lung [6]. Another uses a wire coil to twist and contract the airways [7]. A technique further in the developmental pipeline infuses reagents via a wedged bronchoscope to close the airways with a hydrogel of fibrinogen and thrombin suspensions (fibrin glue) and generate a fibrotic scar in the lung parenchyma. Encouraging phase 2 dose-finding studies have been previously reported in patients with upper-lobe predominant emphysema. Complications included a selflimited inflammatory febrile, dyspnoeic response in most patients, and a few more serious adverse effects, such as pulmonary embolism and pneumonia [8].

In this issue of the European Respiratory Journal, REFAELy et al. [9] report the first use of this method in patients with diffuse, nonupper lobe predominant emphysema. If effective and safe, this would represent a breakthrough for such patients, who are poor candidates for LVRS, and who make up a large fraction of patients with emphysema [1]. The study reports two separate studies that used a low or high dose of reagent in hyperinflated patients with severe, diffuse emphysema as determined by computed tomography (CT) scan. All patients were treated bilaterally in eight lung segments in the upper lobes or lower lobe superior segments, selected by CT and lung perfusion imaging. The low-dose study was terminated after eight subjects due to lack of benefit, and the high-dose arm completed enrolment of 17 subjects.

In the high-dose group, about $60 \%$ of the treated subsegments showed radiological evidence of scarring on CT scan 6 weeks 
after treatment. By 6 months after treatment, the forced expiratory volume in $1 \mathrm{~s}$ (FEV1) improved by a mean of $14 \%$, the Medical Research Council (MRC) dyspnoea score decreased by 0.8 units, and the St Georges Respiratory Questionnaire (SGRQ) decreased by 12 units. Because changes in all of these variables had large standard deviations, the authors also report the percentage of patients showing a minimal clinically significant difference in these variables. At 6 months, $29 \%$ increased $\mathrm{FEV} 1>12 \%, 65 \%$ reduced $\mathrm{MRC}$ dyspnoea $>1$ unit and $77 \%$ improved SGRQ $>4$ units. $12 \%$ increased the 6-min walk $(6 \mathrm{MW})$ by $>50 \mathrm{~m}$, although there were no significant changes in the mean or median 6MW distance. Side-effects were common and consisted of the predictable flu-like reaction, which prolonged hospitalisation, and two exacerbations of chronic obstructive pulmonary disease (COPD) that were considered to be treatment related.

Important questions about these new data include: 1) How does this compare with outcomes from this technique in patients with upper lobe predominant emphysema? 2) How does it compare with results from surgical LVRS? In regards to the first question, the baseline characteristics of patients in the high-dose arm of the current study and that of CRINER et al. [8] were virtually identical. The study designs were also identical, with the exception that CRINER et al. [8] was limited to patients with upper lobe predominant emphysema. Comparing a few of the outcome variables at 6 months among patients treated with the same, high-dose therapy (upper lobe versus diffuse groups), FEV1 increased by $15.6 \%$ versus $13.8 \%$, residual volume/total lung capacity (RV/TLC) decreased by $5.9 \%$ versus $5.4 \%$, the mean improvement in SGRQ was similar (-9.7 versus -12.2$)$ and 6MW distance did not change in either study. Thus, this procedure may offer similar outcomes to patients with either emphysema distribution. This was a result that we had predicted in a theoretical analysis [10], but which has not been the case after surgical LVR $[1,11]$.

Comparing these results with surgical LVR is more problematic. Although many surgeons consider homogeneous emphysema to be unsuitable for LVR, some have reported durable improvements in such patients [11] that are substantially greater than the findings from REFAeLY et al. [9]. It is difficult to assess how the patients may have differed in these series, although the inclusion criteria appear similar. However, surgical morbidity and mortality were also greater than for this bronchoscopic method, and many surgeons do not offer the former procedure to patients with diffuse emphysema.

Other important questions about this technique remain unanswered. One distinct potential disadvantage of biological LVR, when compared with other bronchoscopic LVR techniques, is its irreversible nature. This clinical importance of this issue is currently unknown. Likewise, the safety, especially the risk of rare but serious complications, cannot be evaluated until many more patients are studied. Efficacy questions include finding the best patients, best dose, number of segments, effectiveness of repeated procedures, methods to maximise the percentage of treated segments that shrink, and other variables. Both efficacy and safety metrics require comparison to a sham-treated control group, since motivation can affect many of the subjective outcomes. Blinding may be impossible in light of the inflammatory response to the procedure. Additionally, it is uncertain how much emphasis the Food and Drug Administration (FDA) will place on mortality compared with symptomatic outcomes in their approval deliberations. The rigid study design and timeline required to obtain FDA approval unfortunately conflicts with the spirit of scientific inquiry, making it impossible to follow up fresh ideas or new leads while methods navigate the approval pipeline. If techniques become available, there will be ample opportunity to explore variations of the basic method in a safe and systematic manner. This may identify patient characteristics or technical details that make outcomes better or more consistent.

Finally, armed with all this information, it will fall to patients and their physicians to answer the final question: Is the expected (modest) benefit worth the (modest) risk? Our expectations for surgical LVR have proven to be completely wrong, and we would not venture a guess as to the public acceptance of any given bronchoscopic method. However, what an exciting spectacle it is to witness all these technologies in the Darwinian competition of science and marketing! While some investors may lose, some patients will surely have new options to ponder.

\section{STATEMENT OF INTEREST}

None declared.

\section{REFERENCES}

1 National Emphysema Treatment Trial Research Group. A randomized trial comparing lung volume reduction surgery with medical therapy for severe emphysema. N Engl J Med 2003; 348: 2059-2073.

2 Cardoso PF, Snell GI, Hopkins P, et al. Clinical application of airway bypass with paclitaxel-eluting stents: early results. J Thorac Cardiovasc Surg 2007; 134: 974-981.

3 Wan IY, Toma TP, Geddes DM, et al. Bronchoscopic lung volume reduction for end-stage emphysema: report on the first 98 patients. Chest 2006; 129: 518-526.

4 Sterman DH, Mehta AC, Wood DE, et al. A multicenter pilot study of a bronchial valve for the treatment of severe emphysema. Respiration 2010; 79: 222-233.

5 Aljuri N, Freitag L. Validation and pilot clinical study of a new bronchoscopic method to measure collateral ventilation before endobronchial lung volume reduction. J Appl Physiol 2009; 106: 774-783.

6 Snell GI, Hopkins P, Westall G, et al. A feasibility and safety study of bronchoscopic thermal vapor ablation: a novel emphysema therapy. Ann Thorac Surg 2009; 88: 1993-1998.

7 Herth FJF, Eberhardt R, Ernst A. Pilot study of an improved lung volume reduction coil for the treatment of emphysema. Am J Respir Crit Care Med 2009; 170: A6160.

8 Criner GJ, Pinto-Plata V, Strange C, et al. Biologic lung volume reduction in advanced upper lobe emphysema: phase 2 results. Am J Respir Crit Care Med 2009; 179: 791-798.

9 Refaely Y, Dransfield M, Kramer MR, et al. Biologic lung volume reduction therapy for advanced homogeneous emphysema. Eur Respir J 2010; 36: 20-27.

10 Fessler HE, Permutt S. Lung volume reduction surgery and airflow limitation. Am J Respir Crit Care Med 1998; 157: 715-722.

11 Weder W, Tutic M, Lardinois D, et al. Persistent benefit from lung volume reduction surgery in patients with homogeneous emphysema. Ann Thorac Surg 2009; 87: 229-236. 